\title{
Early Lotus japonicus root transcriptomic responses to symbiotic and pathogenic fungal exudates
}

\author{
Marco Giovannetti ${ }^{1}$, Alfredo Mari, ${ }^{1,2}$, Mara Novero ${ }^{1}$ and Paola Bonfante ${ }^{1 *}$ \\ ${ }^{1}$ Department of Life Science and Systems Biology, Università degli Studi di Torino, Torino, Italy, ${ }^{2}$ Scuola Superiore \\ Sant'Anna di Studi Universitari e Perfezionamento, Pisa, Italy
}

\section{OPEN ACCESS}

Edited by:

Brigitte Mauch-Mani, Université de Neuchâtel, Switzerland

Reviewed by:

Hossein Borhan, Agriculture and Agri-Food Canada,

Canada

Daguang Cai, Christian Albrechts University of Kiel, Germany

*Correspondence: Paola Bonfante, Department of Life Science and Systems Biology, Università degli Studi di Torino, Viale Mattioli 25,

I-10125 Torino, Italy

paola.bonfante@unito.it

Specialty section: This article was submitted to Plant Biotic Interactions, a section of the journal Frontiers in Plant Science

Received: 14 April 2015

Accepted: 15 June 2015

Published: 29 June 2015

Citation:

Giovannetti M, Mari A, Novero M and Bonfante P (2015) Early Lotus japonicus root transcriptomic responses to symbiotic and pathogenic fungal exudates. Front. Plant Sci. 6:480. doi: 10.3389/fp/s.2015.00480
The objective of this study is to evaluate Lotus japonicus transcriptomic responses to arbuscular mycorrhizal (AM) germinated spore exudates (GSEs), responsible for activating nuclear $\mathrm{Ca}^{2+}$ spiking in plant root epidermis. A microarray experiment was performed comparing gene expression in Lotus rootlets treated with GSE or water after 24 and $48 \mathrm{~h}$. The transcriptional pattern of selected genes that resulted to be regulated in the array was further evaluated upon different treatments and timings. In particular, Lotus rootlets were treated with: GSE from the pathogenic fungus Colletotrichum trifolii; short chitin oligomers (COs; acknowledged AM fungal signals) and long COs (as activators of pathogenic responses). This experimental set up has revealed that AM GSE generates a strong transcriptomic response in Lotus roots with an extensive defenserelated response after $24 \mathrm{~h}$ and a subsequent down-regulation after $48 \mathrm{~h}$. A similar subset of defense-related genes resulted to be up-regulated also upon treatment with C. trifolii GSE, although with an opposite trend. Surprisingly, long COs activated both defense-like and symbiosis-related genes. Among the genes regulated in the microarray, promoter-GUS assay showed that LjMATE1 activates in epidermal cells and root hairs.

Keywords: presymbiotic phase, defense response, microarray, Lotus japonicus, germinating spore exudates, chitin oligomers, arbuscular mycorrhizal symbiosis

Abbreviations: CERK1, chitin elicitor receptor kinase 1; COs, chitin oligomers; CSSP, common signaling symbiosis pathway; GSA, glucosidase SA conjugate; GSE, germinating spore exudates; GUS, beta-glucuronidase; LCO, lipo-chitooligosaccharide; LjCCD7, Lotus japonicus carotenoid cleavage dioxygenase 7; LjERF19, Lotus japonicus ethylene responsive factor 19; LjGST, Lotus japonicus glutathione-S-transferase; LjHydr, Lotus japonicus hydrolase; LjLeuc, Lotus japonicus Bark leucoagglutinin precursor; LjMATE1, Lotus japonicus multidrug and toxic compound extrusion protein; LjMTN19, Lotus japonicus medicago truncatula nodulin-like; LjPR10, Lotus japonicus pathogenesis related 10; LjVap-a, Lotus japonicus vapyrin-a; LjVap-b, Lotus japonicus vapyrin-b; LysM, lysin motif; LysM RLK, lysin motif receptor like kinase; MtERF19, Medicago truncatula ethylene responsive factor 19; MtLYK3, Medicago truncatula LysM-type receptor-like kinase 3; MtNFP, Medicago truncatula Nod factor receptor protein; Myc-LCO, mycorrhizal lipo-chitooligosaccharide; nsMyc-LCOs, non-sulphated mycorrhizal lipo-chitooligosaccharide; NSP1, nodulation signaling pathway 1; NSP2, nodulation signaling pathway 2; PAMP, pathogenassociated molecular pattern; PTI, PAMP-triggered immunity; RAM, required for arbuscular mycorrhization; ROS, reactive oxygen species; SA, salicylic acid; sMyc-LCO, sulphated mycorrhizal lipo-chitooligosaccharide. 


\section{Introduction}

Since the land conquest about 450 million years ago, plants had to deal and relate with both pathogenic and beneficial organisms. On the one hand, plants had to protect themselves from pathogens, evolving strong defense mechanisms to effectively ward them off (Jones and Dangl, 2006). On the other hand, plants have developed symbiotic relationships based on a fair nutrient exchange (Kiers et al., 2011). Understanding how plants can discriminate between friends and foes is a crucial question in plant biology (Hayashi and Parniske, 2014; Bonfante and Genre, 2015) with a direct effect on agricultural practices. On the pathogen side, a big effort has been invested in the study of fungal and bacterial effectors and plant receptors, focusing on the modulation of plant immunity, plant disease resistance and its application in modern agriculture (Dodds and Rathjen, 2010; Macho and Zipfel, 2014).

Among the beneficial microorganisms capable to form symbiosis with plants, research has mainly been guided by nutritional aspects (Gutjahr and Parniske, 2013) with less focus on immunity and compatibility aspects (Rey and Schornack, 2013). The two most studied symbioses between plants and soil microorganisms are symbiotic nitrogen fixation (Gage, 2004) and arbuscular mycorrhizal (AM; Parniske, 2008). Plant genetics and mutant analyses allowed to characterize several genes required for both root endosymbioses (in primis LjSYMRK/DMI2, LjPOLLUX/DMI1, LjCCaMK/DMI3 in the case of the model legumes Lotus japonicus and Medicago truncatula, respectively), thus defining a CSSP (Oldroyd, 2013).

Knowledge on AM signals perception is not yet fully understood: LCOs have been identified in AM roots and in germinating AM spore exudates using the same bioassay that was set up for Nod factor characterization and named Myc-LCO (Maillet et al., 2011). Myc-LCO can induce lateral root formation, calcium spiking and a huge set of gene regulation but all of these actions are dependent on the MtNFP, the Nod Factor receptor (Maillet et al., 2011; Czaja et al., 2012; Genre et al., 2013). By contrast, it has been demonstrated that tetra- and pentamers of $\mathrm{N}$-acetylglucosamine (chitin oligosaccharides: CO4, CO5), contained in spore GSE, activate the CSSP independently of MtNFP (Chabaud et al., 2011; Genre et al., 2013). Moreover CO4 and $\mathrm{CO} 5$ concentration is strongly increased when germinating spores are treated with GR24, a synthetic strigolactone analog. The same molecules have been shown to be active also in rice (Sun et al., 2015) and carrot, but not in Arabidopsis (Genre et al., 2013).

Plant-microbe interactions utilize similar chemical signatures to mediate biological processes leading to a symbiotic or a pathogenic relationship (Liang et al., 2013; Evangelisti et al., 2014; Miyata et al., 2014; Wang et al., 2014; Zhang et al., 2015). For example, a cross-talk between the CSSP and chitindefense responses has been suggested: MtNFP, involved in the formation of endosymbioses, has been shown to be unsuspectedly involved in plant immunity, thus $m t n f p$ plants resulted to be more susceptible to pathogens, such as Aphanomyces euteiches and Colletotrichum trifolii, and its overexpression increased resistance
(Gough and Jacquet, 2013; Rey et al., 2013). Another hint about the existence of parallels between Nod factor-induced and chitininduced signaling, mediated by the respective LysM RLK, is given by the similarity of Nicotiana benthamiana responses to MtNFP and MtLYK3 (both part of the Nod factor receptor complex) co-production and AtCERK1 production (Pietraszewska-Bogiel et al., 2013). It was recently shown that OsCERK1, known to be responsible of the detection of pathogenic chitin molecules in rice, is also involved in the interaction with AM fungi (Miyata et al., 2014; Zhang et al., 2015).

Both the Nod Factor receptor and AtCerk1 and, probably, the still unknown Myc-LCO receptor(s), bind to chitin residues, but the presence of different receptor complexes seems to allow a correct recognition of different chitin molecules and a discrimination between symbiont and pathogen chitin signals (Antolín-Llovera et al., 2014; Cao et al., 2014; Ried et al., 2014).

Fully colonized and functional AM roots were extensively studied by expression profiling, initially with a whole organ approach (Fiorilli et al., 2009; Guether et al., 2009), then through cell-type specific microarray (Hogekamp et al., 2011; Gaude et al., 2012), and more recently with RNA-seq approaches (Ruzicka et al., 2013; Handa et al., 2015). But up to date, genome-wide studies analyzing the presymbiotic stages only considered the transcriptomic impact of Myc-LCOs (Czaja et al., 2012) or the stage of hyphopodium formation (Hogekamp and Küster, 2013). The goal of this investigation is to characterize the transcriptome of L. japonicus upon perception of Gigaspora margarita GSE. GSE may contain not only a mix of simple sulfated and non-sulfated LCOs (referred to as sMyc- and nsMyc-LCOs; Maillet et al., 2011), COs (Genre et al., 2013), and effectors (Kloppholz et al., 2011; Tisserant et al., 2013) - each one probably playing a role during AM presymbiotic phase (Sun et al., 2015) - but also - still unknown - fungal molecules perceived by plants. This exudate could represent an ideal mix to investigate plant responses to AM fungi, since it better mimics the bioactive molecules released by AM fungi in natural conditions during plant-fungal presymbiotic interaction and defense-like responses. As a second goal, we wanted to go deeper in the characterization of plant-defense genes - which are known to be activated by AM fungi not only in roots but also in other organs like shoots and fruits (Fiorilli et al., 2009; Zouari et al., 2014) - in order to understand whether AM exudates may simultaneously activate both symbiotic and pathogenic-like responses. To validate this hypothesis, we treated Lotus seedlings with short (CO5) and long (CO8) COs, since the first elicit the symbiotic calcium spiking (Genre et al., 2013), while $\mathrm{CO} 8$ are the chitooligosaccharides which act as elicitors of defense (Hayafune et al., 2014). In parallel, we tested the specificity of a subset of genes by treating Lotus rootlets with GSE from a pathogen fungus such as $C$. trifolii.

Our genome-wide expression analysis revealed: (i) more than 100 genes induced by the perception of fungal GSE; (ii) a wide and extensive defense-like response in Lotus root $24 \mathrm{~h}$ after the perception, and a subsequent down-regulation of defense-like genes after $48 \mathrm{~h}$, (iii) the activation by $\mathrm{CO} 8$ of both defense-like and symbiosis-like genes, (iv) similarities between the symbiotic and pathogenic signature elicited by the AM and C. trifolii GSE, 
and (v) the localization of one of the activated genes in epidermal cells by means of promoter-GUS assays.

\section{Results}

\section{G. margarita GSE Triggers a Specific Gene Expression}

To record transcriptional responses toward symbiotic signals, we treated L. japonicus wild-type roots with G. margarita GSE. A microarray experiment with RNA coming from Lotus rootlets treated with GSE allowed us identifying 134 genes differently regulated after $24 \mathrm{~h}$ of treatment with GSE and 21 genes after $48 \mathrm{~h}$ (Figure 1). One third of the genes resulted to be linked to defense or redox mechanisms: they showed an up-regulation after $24 \mathrm{~h}$ and most of them had a dramatic down-regulation after $48 \mathrm{~h}$ (Table 1), probably pointing to a defense-like response of the plant to the AM fungal exudates and a subsequent downregulation of that response (data sheet 1 ).

Among the most up-regulated genes at $24 \mathrm{~h}$, Table 2 shows a pathogenesis-related protein, a lipase-hydrolase protein and various glutathione- $S$-transferases (GSTs), revealing a response of the root to microbe signals in line with responses already registered in plant root hairs upon early infection by symbiotic soil bacteria (Libault et al., 2010; Gourion et al., 2015). Among the most down-regulated genes, we identified genes linked to the

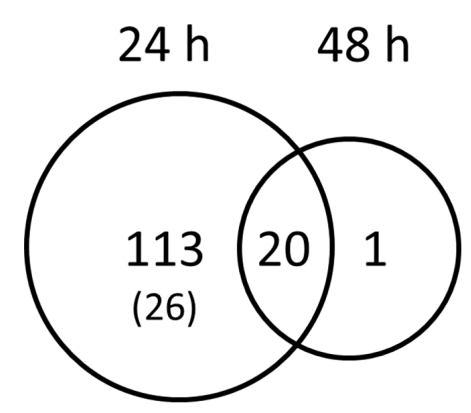

FIGURE 1 | Genes specifically activated by Gigaspora margarita spore germination water. The Venn diagram visualize the coactivation of Lotus japonicus wild-type root genes by GSE 24 and $48 \mathrm{~h}$ after treatment. Numbers indicate the number of genes with FDR values $<0.05$ and a fold change cut-off $<-1$ or $>+1$. The number into brackets indicate the genes that resulted to be down-regulated.

TABLE 1 | List of gene categories regulated in the microarray experiment.

\begin{tabular}{lc}
\hline Putative function & \# of genes \\
\hline Redox related genes & 35 \\
Defense/stress related genes & 19 \\
Hormone related genes & 7 \\
Transporters & 6 \\
Signaling related genes & 5 \\
Secondary metabolism & 8 \\
Other functions & 54 \\
& \multirow{2}{*}{134}
\end{tabular}

phospholipid metabolism, such as a putative phosphatidylinositol phosphatidylcholine transfer protein, and to the ion traffic.

\section{AM-GSE Activate Genes in an Opposite Way than Pathogen GSE}

Looking at the relevant Lotus defense responses elicited by the GSE (one third of the differentially expressed genes were related to defense) we wondered whether this gene subset represented a part of the genes required for the establishment of AM symbiosis or whether it mirrored mostly a defense response, similar to the one elicited by pathogenic fungi.

To better understand this point, from the microarray data we selected four genes that were strongly down-regulated between 24 and $48 \mathrm{~h}$, one gene that resulted to be up-regulated at both time points and one putative marker gene (LjERF19). We compared the relative expression of these six genes over two time points ( 24 and $48 \mathrm{~h}$ ) with the gene induction generated by spore germination exudates from a fungal pathogen, C. trifolii. This biotrophic pathogen is known to trigger early cell responses in the root epidermis (Genre et al., 2009), but its GSE (which contains COs of variable length) cannot activate the CSSP (Genre et al., 2013). Figure 2 shows that four out of five selected genes (a protein inhibitor-LjPI, a hydrolase-LjHydr, a pathogenesis related protein-LjPR10 and a lectin-like proteins-LjLeuc) when treated with $G$. margarita GSE are highly overexpressed at $24 \mathrm{~h}$ and then dramatically down-regulated, confirming the data coming from the microarray. By contrast, the treatment with C. trifolii GSE resulted in an opposite behavior, showing a slower increase of expression between 24 and $48 \mathrm{~h}$. The only exception was represented by $L j P R 10$ that was downregulated with a similar expression pattern than AM fungal exudates.

Finally we checked for the trend of a LjGST, one of the few genes that in the microarray was overexpressed at both time points. QPCR confirmed this trend with AM GSE treatment but the transcript was not detectable after treatment with pathogenic GSE. LjGST function within AM development is still unknown but the gene is overexpressed also in later stage of symbiosis (Wulf et al., 2003; Hao et al., 2012; Hogekamp and Küster, 2013).

In addition, to understand if GSE could contain not only bio-active molecules involved in signaling, but also effectors down-regulating host defense pathways, we checked for the expression level of LjERF19. LjERF19 is the Lotus ortholog of $M t E R F 19$, a transcription factor targeted and down-regulated by AM fungal effector SP7 (Kloppholz et al., 2011). As reported for Medicago, we show a down-regulation of the transcription factor from 24 to $48 \mathrm{~h}$ after AM but not C. trifolii GSE treatment. This result can indirectly show the presence of effectors in G. margarita GSE, specifically targeting LjERF19.

Taken in the whole, the qRT-PCR experiment suggests that the plant genes regulated by pathogenic or symbiotic signals are largely overlapping with a specific trend of expression. The results therefore suggest a possible role represented by the timing of the gene activation that could be responsible of triggering different physiological plant responses. 
TABLE 2 | Most up- and down- regulated genes in Lotus japonicus wild-type roots treated with Gigaspora margarita GSE.

\begin{tabular}{|c|c|c|c|c|c|}
\hline \multirow[t]{2}{*}{ Lotus GeneChip ID } & \multirow[t]{2}{*}{ Annotation } & \multicolumn{2}{|c|}{ Log2 FC } & \multicolumn{2}{|c|}{$p$-values } \\
\hline & & $24 \mathrm{~h}$ & $48 \mathrm{~h}$ & $24 \mathrm{~h}$ & $48 \mathrm{~h}$ \\
\hline Ljwgs_075692.1.1_at & Lipase hydrolase-like protein & 4.92 & -0.52 & 0.000 & 0.369 \\
\hline Ljwgs_079986.1_s_at & PR10-1 protein & 4.82 & 0.73 & 0.000 & 0.205 \\
\hline Ljwgs_044810.1_at & Bark leucoagglutinin I precursor & 4.57 & 0.77 & 0.000 & 0.334 \\
\hline TM1656.16_at & Pectinesterase & 4.22 & -0.17 & 0.000 & 0.808 \\
\hline Ljwgs_051780.1_at & UDP-glucose:SA glucosyltransferase & 3.96 & 4.60 & 0.000 & 0.000 \\
\hline chr1.CM0064.61_s_at & Germin-like protein & 3.95 & 2.23 & 0.000 & 0.006 \\
\hline Ljwgs_028218.2_at & Glutathione S-transferase GST 9 & 3.90 & 3.50 & 0.000 & 0.000 \\
\hline Ljwgs_075865.1_at & Bark agglutinin I, polypeptide B precursor & 3.77 & 0.83 & 0.000 & 0.200 \\
\hline chr6.CM0437.7_at & MTN19 gene precursor & 3.21 & 1.56 & 0.000 & 0.016 \\
\hline chr5.CM0909.45_at & Glutathione-S-transferase GST 15 & 3.15 & 3.36 & 0.000 & 0.000 \\
\hline chr3.CM0208.34_at & Putative Fe(II) ascorbate oxidase & 3.10 & 0.12 & 0.000 & 0.740 \\
\hline chr2.CM0201.55_at & Auxin-induced protein & 3.08 & 3.96 & 0.000 & 0.000 \\
\hline chr4.CM0046.42_at & Glutathione-S-transferase GST 14 & 3.02 & 2.36 & 0.000 & 0.000 \\
\hline chr1.CM0010.42_at & Purple acid phosphatase (PAP22) & -1.52 & -0.25 & 0.000 & 0.300 \\
\hline Ljwgs_066244.1_at & Putative glucanase & -1.74 & -0.10 & 0.000 & 0.689 \\
\hline chr4.CM0126.67_at & Hypothetical protein & -1.80 & -0.25 & 0.000 & 0.422 \\
\hline Ljwgs_122957.1_at & Cyclic nucleotide and calmodulin-regulated ion channel-like protein & -1.83 & -0.24 & 0.000 & 0.406 \\
\hline Ljwgs_025743.1_s_at & Cyclic nucleotide and calmodulin-regulated ion channel-like protein & -1.83 & -0.22 & 0.000 & 0.393 \\
\hline Ljwgs_071032.1_s_at & SEC14 - like protein & -1.88 & -0.19 & 0.000 & 0.509 \\
\hline chr4.CM0399.52.1_at & AP2 domain containing protein RAP2.11 & -1.91 & 0.01 & 0.000 & 0.979 \\
\hline Ljwgs_007118.2_at & Unknown protein & -1.93 & -0.25 & 0.000 & 0.412 \\
\hline chr5.TM1125.10_at & Glutathione peroxidase -like protein & -2.11 & -0.03 & 0.000 & 0.925 \\
\hline Ljwgs_071601.1_s_at & Putative phosphatidylinositol phophatidylcholine transfer protein & -2.19 & -0.15 & 0.000 & 0.587 \\
\hline
\end{tabular}

Gene expression is represented by Log2-fold change values for the two treatments (24 and $48 \mathrm{~h}$ after GSE application), indicating also the $p$-values.

\section{Short and Long Chitin Oligomers Elicit Different Gene Expression}

On the basis of insights on the composition of active and nonactive chitin-related oligomers contained in AM and C. trifolii exudates (Genre et al., 2013), and our RT-PCR experiments (Figure 2), we wondered whether short (CO5) and long (CO8) COs could partly mimic symbiotic and pathogenic signals, respectively. It is known that $\mathrm{CO} 5$ are able to induce $\mathrm{Ca}^{2+}$ spiking (Genre et al., 2013), while CO8 which miss to elicit the symbiotic-calcium spiking, can be associated to the defenseresponses previously identified (Liang et al., 2014). Moreover we hypothesized that G. margarita GSE could contain a variety of chitin molecules and therefore able to trigger both symbiosis and pathogenic related genes. To test this, we performed a set of qPCR on Lotus rootlets treated with water, short (CO5), long (CO8) COs, and G. margarita GSE focusing on an earlier time point, $1 \mathrm{~h}$ after treatment, in order to be closer to the calcium spiking events (Figure 3). We chose some defense-related genes activated in the microarray and some other genes that could be considered gene marker of AM development. The treatment inducing the highest regulation of genes was GSE. Consistently with our prediction, defense-like genes, such as LjPR10, LjLeuc and LjMATE1, resulted also to be induced both by $\mathrm{CO} 8$ and GSE but not by water and CO5 treatment. Surprisingly, CO8 were also able to induce genes involved in mycorrhizal colonization, such as vapyrin genes
(LjVap-a and LjVap-b; Figure 3), regardless of the fact that the $\mathrm{CO} 8$ are not able to elicit a nuclear calcium spiking. At the same time, CO8 can activate a key gene in the metabolic pathway of strigolactone synthesis (LjCCD7). Overall, CO5 were less active than CO8 with the exception of the positive regulation of LjERF19, the ortholog of MtERF19 (Kloppholz et al., 2011).

\section{Promoter GUS Localization}

To localize Lotus transcriptomic responses to GSE, we focused on LjMATE1, a multidrug and toxic compound extrusion protein that resulted to be up-regulated in the microarray experiment (data sheet 1 ) and is potentially involved in different kind of cellular detoxification. The same gene is involved in citrate transfer within nodules (Takanashi et al., 2013) and upregulated in mycorrhizal roots (Handa et al., 2015) therefore making it a good candidate for a putative function in legume endosymbioses. The putative promoter region of LjMATE1, 2243 bp upstream of the coding region, were fused to the reporter gene GUS. This construct was introduced into Lotus roots by Agrobacterium rhizogenes-mediated transformation. Composite plants were grown in plates, transgenic hairy roots were generated and treated after 4 weeks with long COs (CO8) for $1 \mathrm{~h}$. Roots incubated with GUS buffer overnight at $37^{\circ} \mathrm{C}$ showed a typical epidermal coloration (Figure 4A) with homogenous blue color coming from root hairs (detail showed in Figure 4B) and outer 


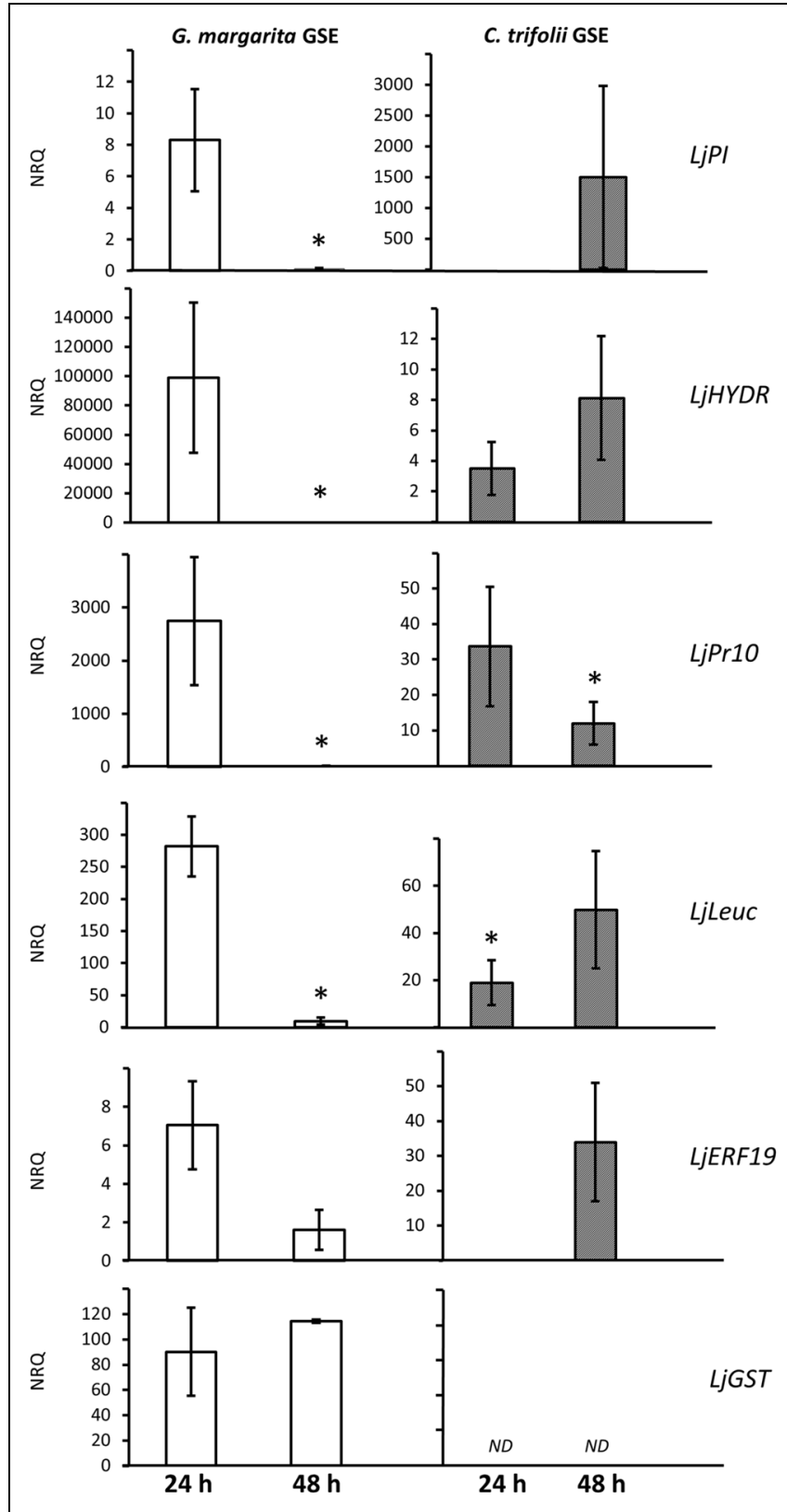

FIGURE 2 | Comparison of gene induction by G. margarita or C. trifolii GSE 24 and $\mathbf{4 8 ~} \mathbf{h}$ after the treatment. Putative defense-like genes were selected from the microarray list and their gene expression tested in a new experiment with also germination water from a fungal pathogen, Colletotrichum trifolii, after 24 and $48 \mathrm{~h}$. Bars represent the mean of four biological replicates $\pm \mathrm{SE}$. Bars subtended by the asterisk differ significantly at $p$-value $<0.05$, according to the Kruskal-Wallis test. NRQ stands for Normalized Relative Quantities. LjPI, proteinase inhibitor; LjHYDR, hydrolase-like protein; LjPR10, pathogenesis related protein; LjLeuc, Bark leucoagglutinin precursor; LjERF19, ethylene responsive element; LjGST, glutathione-S-transferase.

root cell layers. In conclusion it seems that the localization pattern of LjMATE1 is consistent with the functionality of a gene hypothetically involved in the presymbiotic fungal-plant interaction.

\section{Discussion}

The combination of untargeted and targeted transcriptomic analyses has allowed us to add some novel information to the still opened question of plant host responses to AM signals during the presymbiotic phases. In addition, the comparison of the effects elicited by exudates released by symbiotic and pathogenic fungi has revealed how the host plant responds activating the same gene subset but with a specific timing.

\section{G. margarita GSE Trigger L. japonicus Transcriptomic Responses}

The results of our experiment (the use of the whole GSE which contains a cocktail of known and unknown molecules) led to a first conclusion: G. margarita GSE-induced gene regulation in Lotus is highly different than gene expression induced in Medicago by purified Myc-LCO (Czaja et al., 2012). Among the 134 genes regulated by GSE in Lotus, just two genes show a significant similarity with Medicago genes induced by Myc-LCO treatment, a putative GST (Mtr.18369.1.S1_at) and a putative endoglucanase (Mtr.50565.1.S1_at). These few overlaps could be due to the different biological system or to the different activity exerted by the complexity of GSE.

The induction of gene expression resulted to be mostly transient, consistently with the results obtained with MycLCOs and differently from gene regulation by purified NodLCOs (Czaja et al., 2012). However, we cannot exclude that the transiency of the response is due to instable nature of AM fungal molecules or, alternatively, to the presence in the GSE of enzymes or other molecules able to inactivate or outcompete for fungal molecules, as it happens with Cladosporium fulvum LysM effectors (Sanchez-Vallet et al., 2013). This transiency is well indicated by the low number of regulated genes and the overall highest $p$-value at $48 \mathrm{~h}$. Nor the genes encoding components of the CSSP did show a marked expression change toward GSE, or the genes encoding GRAS transcription factors acting downstream of $\mathrm{Ca}^{2+}$ signaling, such as NSP1, NSP2, RAM1 (Gobbato et al., 2012), RAM2 (Wang et al., 2012), or DELLA (Floss et al., 2013): however, it is worth to highlight the fact that all of these genes were identified and characterized through forward genetics and mutant phenotyping and their gene regulation during presymbiotic phase was not described elsewhere.

Among the regulated genes, GSTs represents a major group of detoxification enzymes, regulated in vivo by ROS. ROS production at the infection site is the earliest response of PAMPtriggered immunity (PTI). Apart from primary effects, such as cell wall strengthening and induction of antimicrobial activity, ROS function as diffusible second messenger, inducing several resistance responses including synthesis of pathogenesis-related proteins (Zurbriggen et al., 2010), which have been recently demonstrated to be relevant also for symbiotic interaction (Gourion et al., 2015). Other species of oxidative burst are demonstrated to be involved in AM symbiosis as nitric oxide accumulation (Calcagno et al., 2012) and in nodules (Marino et al., 2011). 


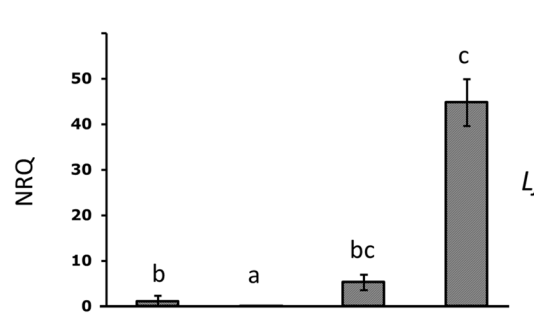

LjPR10
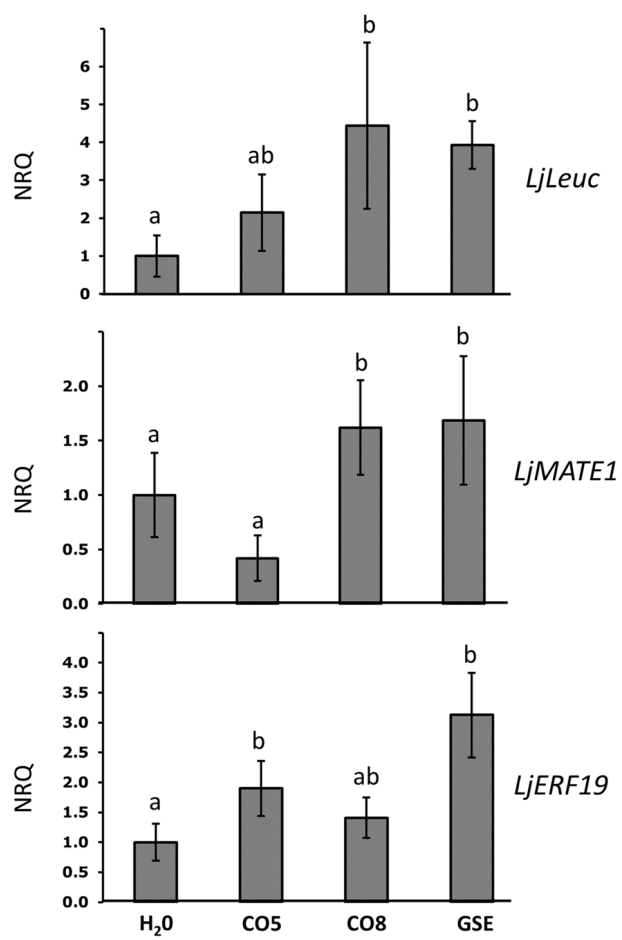

FIGURE 3 | Relative expression of symbiotic and defense-like genes after treatment with water, short (CO5), long (CO8) chitin oligosaccharides and G. margarita GSE, $1 \mathrm{~h}$ after the treatment. The expression of genes previously characterized as related to defense or symbiosis was characterized after treatment of Lotus rootlets with short or long chitin oligosaccharides. As controls we used G. margarita GSE and water. Bars represent the mean of four biological replicates $\pm S E$. Bars

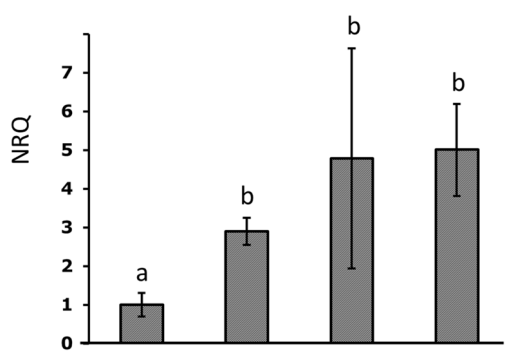

LjVap-a
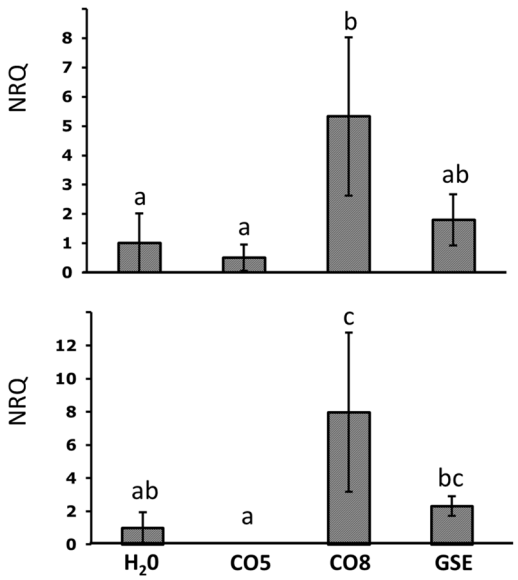

LjCCD7

LjVap-b

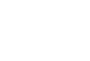

subtended by the same low-case letter do not differ significantly at $p$-value $<0.05$, according to the Kruskal-Wallis test and Mann-Whitney Post hoc test. NRQ stands for Normalized Relative Quantities. LjPR10, pathogenesis related protein; LjLeuc, Bark leucoagglutinin precursor; LjMATE1, multidrug and toxic compound extrusion gene; LjERF19, ethylene responsive element 19; LjVap, vapyrin-like gene; LjCCD7, carotenoid cleavage dioxygenase 7 .

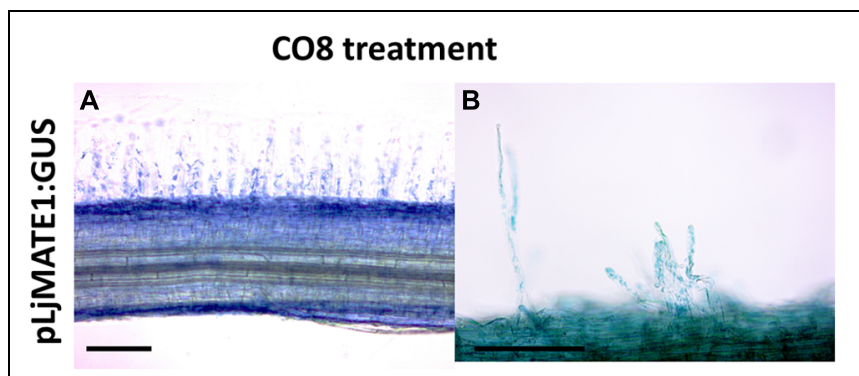

FIGURE 4 | Histochemical GUS staining of $L$. japonicus roots expressing pLjMATE1:GUS. After treatment with long COs (CO8), L. japonicus roots expressing pLjMATE1:GUS shows a blue coloration that seems to regard exclusively epidermal cells (A) as hinted by the typical blue signal coming from root hairs (B). Cortical and central cylinder cells are not marked. Scale bars corresponds to $100 \mu \mathrm{m}$.
Lotus japonicus medicago truncatula nodulin-like 19 (LjMTN19) seems to be a possible key gene in both nodule and AM formation since its expression is induced both during mature AM colonization in Lotus (Guether et al., 2009) and during nodule formation (Moreau et al., 2011; Naya et al., 2014). Its biological role and function is still unknown but it was shown that Mtn19-like gene from pea increases also in pods treated with the insect elicitor Bruchin B (Doss, 2005) and thus it has been proposed to be involved in plant biotic responses. Our findings support this hypothesis also for Lotus.

Overall many of the genes that showed a highest regulation were previously characterized as involved in plant-microbe interaction: in virus-tobacco interaction, salicylic acid (SA) can activate plant resistance and its levels increase systematically following the hypersensitive response. The SA increase in the inoculated leaf coincided with the appearance of a GSA thanks 
to the higher activity of UDP-glucose: SA glucosyltransferase (Enyedi and Raskin, 1993). The same class of genes resulted to be regulated in our set up but also involved in Arabidopsis resistance against Pseudomonas syringae (Boachon et al., 2014).

Altogether novel genes activated by AM fungal exudates have been identified and could constitute a target for future analyses.

\section{A Cocktail of Specific and Non-Specific Responses}

Due to the fact that the majority of genes regulated in the microarray seems not to be specific of AM interactions but belonging to generic plant responses to biotic stress, we wanted to verify whether the same genes could be induced upon contact with GSE from C. trifolii, a pathogenic fungus. This experiment allowed to show that most of the genes were still activated but with a completely different pattern of expression: a gradual overexpression over time as opposed to a dramatic down-regulation happening after AM fungal GSE treatment (Figure 2). It would be challenging to demonstrate that this expression pattern could mirror the action of effectors probably contained in the GSE. The genome sequence of $R$. irregularis has revealed the presence of hundreds of small secreted proteins (Tisserant et al., 2013). The fungus we used is G. margarita which is, among Glomeromycota, phylogenetically quite far but we expect biological tools necessary to form a symbiosis to be well conserved among AM fungi.

As a further step we wondered whether we could correlate the activity of some chitin oligosaccharides, demonstrated to be present in the GSE, to the different genes activated and, eventually, discriminate between symbiotic and defense-like responses. Genre et al. (2013) showed that $\mathrm{CO} 4$ and $\mathrm{CO} 5$ are responsible of calcium spiking in transformed roots of Medicago, in contrast with longer chitin oligosaccharides such as CO8. Therefore our hypothesis was that long chitin oligosaccharides could be more connected with plant defense response whereas shorter oligomers could be able to trigger mostly genes related to symbiotic pathway or eventually counteract plant immunity system as suggested by (Liang et al., 2014). Comparing our results with results from Czaja et al. (2012) it is worth to highlight the fact that long COs, such as $\mathrm{CO} 8$, are sufficient to activate genes essential for the molecular dialog, like the strigolactones (Bonfante and Genre, 2015), as well as genes which allow an efficient epidermal penetration by AM fungi and arbuscule formation, such as Vapyrin (Pumplin et al., 2009; Figures 3 and 5). These results could lead to two different conclusions: AM fungal exudates can contain longer $\mathrm{COs}$ with a "symbiotic" function in plant-fungus chemical communication. As an alternative, longer COs, associated with pathogen-like organisms, can activate genes characterized as mycorrhizal specific, as Vapyrin, but with other unknown functions in different plant-microbe interaction, in line with what was shown for $M$. truncatula symbiosis mutants ( $m t n f p$ and $m t l y k 3$, for example) that were affected in the interaction with a biotrophic root pathogen (Rey et al., 2015). To delve into expression pattern of genes regulated in the array and to develop a possible tool and marker of GSE activity, we obtained promoter-GUS lines of one gene of interest. As shown in Figure 3, a clear blue coloration

\begin{tabular}{lccccc}
\hline & \multicolumn{4}{c}{ treatment } \\
\cline { 2 - 5 } & G. m. GSE & C. $t . \mathrm{GSE}$ & $\mathrm{CO5}$ & $\mathrm{CO} 8$ \\
\hline Defence-related genes & $\searrow$ & $\nearrow$ & + & + \\
Symbiosis-related genes & + & n. d. & + & + \\
$\mathrm{Ca}^{2+}$ spiking(Gerre etal, 2013) & + & - & + & - \\
\hline
\end{tabular}

FIGURE 5 | Activity of fungal exudates and COs. G. margarita GSE (G. $m$. GSE) and C. trifolii exudates (C. t. GSE) activate a similar set of

defense-related genes in $L$. japonicus rootlets, but with a different timing of the expression profile: from high to low (G. m. GSE) and from low to high (C. $t$. GSE), as shown by arrow direction. A subset of these genes was shown to be induced also upon treatment with short (CO5) or long (CO8) COs, $1 \mathrm{~h}$ after treatment. Symbiosis-related genes were up-regulated by G. $m$. GSE, as expected, but their expression was not discriminated by $\mathrm{CO} 5$ and $\mathrm{CO} 8$ treatments, differently from calcium spiking activity. This test seems to represent up-to-date the only reliable marker of the presymbiotic phase.

confirmed the expression of the gene LjMATE1 in epidermal cells and root hairs, as we could expect from genes involved in the pre-symbiotic plant-fungal interactions. Promoter-GUS experiment showed that $L j M A T E 1$ is activated in trichoblasts. It is known that $\mathrm{Ca}^{2+}$ spiking mainly regards atrichoblasts and AM fungi tend to avoid roots with extensive root hairs, by contrast, it seems that at least one of the genes whose expression is induced upon exposure with fungal exudates, localize at trichoblast level (Figure 3), thus providing further evidence of the fact that these response do not overlap with the CSSP. Interestingly LjMATE1 is also involved in nodule maintenance, therefore potentially conserved among the two endosymbioses. In root nodule symbiosis, it was shown to be a citrate transporter and its silencing altered Fe localization in mature nodules of L. japonicus (Takanashi et al., 2013; González-Guerrero et al., 2014).

Taken in the whole, our experiments have provided a set of genes which are regulated by the AM signals, have revealed that plant responses are a mix of specific and generic responses and that molecules like $\mathrm{CO} 5$, eventhough involved in AM symbiosis, are not strongly involved in transcriptional responses. By contrast, $\mathrm{CO} 8$ activate AM symbiotic-related genes, irrespectively, of being traditionally associated to defenseresponses. However, irrespectively, of such differences, among the activated genes, LjMATE1 results to be expressed at the plant epidermis providing new tools to investigate the molecular dialog between fungi and their host plants.

\section{Materials and Methods}

\section{Plant and Fungal Materials}

Lotus japonicus (Regel) K. Larsen seeds (MG20, WT) were scarified and surface-sterilized for $5 \mathrm{~min}$ in concentrated sulfuric acid and washed three times with sterile water. In a second step the seeds were incubated for $3 \mathrm{~min}$ in 1:3 diluted commercial bleach with 1:1000 Triton-X. After washing three times with sterile water, the seeds were germinated on water-agar $(0.6 \%)$ in Petri dishes. For the microarray experiment, three seedlings were left in a $1.5 \mathrm{~mL}$ eppendorf tube containing $1 \mathrm{~mL}$ of $10 \mathrm{X}$ 
GSE (Genre et al., 2013) or water for 24 and 48 h. C. trifolii race 2 strain MUT 3930 (Richard O'Connell, BIOGER-CPP, 78850 Thiverval-Grignon, France) conidia were produced after 7 days at $23^{\circ} \mathrm{C}$ on a modified Mathur's medium $(0.1 \%$ yeast extract; $0.1 \%$ BactoPeptone; $1 \%$ sucrose; $0.25 \% \mathrm{MgSO}_{4} 7 \mathrm{H}_{2} \mathrm{O}$; $0.27 \% \mathrm{KH}_{2} \mathrm{PO}_{4} ; 2 \%$ agar in 11 of sterile distilled water). Spores were prepared as previously described (Torregrosa et al., 2004). A total of $10^{7}$ spores were diluted in $100 \mathrm{ml}$ of sterile $\mathrm{H}_{2} \mathrm{O}$. After $24 \mathrm{~h}$ incubation at $24^{\circ} \mathrm{C}$, the germinated spores were pelleted by centrifugation at $5000 \mathrm{~g}$ for $15 \mathrm{~min}$ and the GSE was recovered for analysis. C. trifolii GSEs were lyophilized and suspended in $1 \mathrm{~mL}$ of $\mathrm{H}_{2} \mathrm{O}$.

\section{RNA Isolation and Microarray Hybridization}

Roots were harvested and immediately frozen in liquid nitrogen in a $2 \mathrm{ml}$ reaction tube. Two clean metal balls were added into every tube and frozen again. Plant material was then ground using a Retsch ${ }^{\circledR}$ ball mill for $2 \mathrm{~min}$, at least three biological replicate per each condition. RNA was extracted using a modified 'pine-tree-method' (Guether et al., 2009). Integrity of RNA samples was checked using an Agilent 2100 Bioanalyzer. RNA purity was determined by ensuring spectrophotometric ratios of A260nm/A280nm $\sim 2$ and A260nm/A230nm $\geq 2$. Removal of genomic DNA was done using the Turbo DNAfree $^{\mathrm{TM}}$ reagent (Ambion, Austin, TX, USA) following the manufacturer's instructions. Absence of genomic DNA was verified by RT-PCR with intron-specific primer for tubulin $\beta-5$ (TM0371b.4/TC18284). For each sample, $1 \mu \mathrm{g}$ of total RNA was send to AtlasBiolabs ${ }^{1}$ to perform the microarray experiment. cRNA was hybridized to the Genechip ${ }^{\circledR}$ Lotus1a520343 and scanned, according to the manufacturer's instructions.

\section{Data Analysis}

Microarray data were analyzed using the bioconductor software package for the $\mathrm{R}$ programming language (Gentleman et al., 2004). Data quality was assessed using the AffyPLM packages

${ }^{1}$ www.atlas-biolabs.de
(Gautier et al., 2004), and expression estimates were obtained using the RMA algorithm (Irizarry et al., 2003). Control and bacterial probe-sets were removed, and only genes assigned as present $(P<0.05)$ using the MAS5 present/absent algorithm were retained. Statistical testing for differential expression was performed using mixed models with the LIMMA bioconductor package (Smyth, 2004). Comparison of the obtained data sets to the previous published microarray studies were based on TBLASTX. For the L. japonicus data sets (Deguchi et al., 2007) an e-value threshold of 1e-50 was applied. Furthermore, microarray data are available in the ArrayExpress database ${ }^{2}$ under accession number E-MTAB-3119.

\section{cDNA Synthesis and Real Time RT-PCR}

Real-time experiments were carried out on material derived from root. cDNA synthesis was performed using SuperScriptII ${ }^{\circledR}$ Reverse Transcriptase and $1 \mu \mathrm{g}$ of total RNA, following the protocol of the supplier (Invitrogen Ltd, Paisley, UK). Oligonucleotide sequences of all the primers are listed in Table 2. Quantitative RT-PCR was carried out with an iCycler apparatus (Bio-Rad Laboratories, Hercules, CA, USA). Each PCR reaction was carried out in a total volume of $15 \mu$ l. The following PCR program was used: $95^{\circ} \mathrm{C}$ for $90 \mathrm{~s}, 40$ cycles of $95^{\circ} \mathrm{C}$ for $15 \mathrm{~s}, 60^{\circ} \mathrm{C}$ for $30 \mathrm{~s}$. A melting curve $(54-70$ steps with a heating rate of $0.5^{\circ} \mathrm{C}$ per $10 \mathrm{~s}$ and a continuous fluorescence measurement) was recorded at the end of every run to exclude primers generating non-specific PCR products (Ririe et al., 1997). All reactions were performed for at least three biological and two technical replicates. Baseline range and CT values were automatically calculated using the iCycler software. In order to compare data from different PCR runs or cDNA samples, CT values of all genes were normalized to the CT value of UBQ10 (chr1.TM0487.4) as previously described (Guether et al., 2009). Table 3 indicates the list of primers used in this experiment. Handling small sample size, statistical differences for QPCR analysis were calculated by Kruskal-Wallis

${ }^{2}$ www.ebi.ac.uk/arrayexpress

TABLE 3 | List of primers used in this study.

\begin{tabular}{llll}
\hline Gene locus & Gene name & Primer forward & Primer reverse \\
\hline Ljwgs_079986.1 & LjPR10 & CTAAAGGTGATGCTAAACCC & GCAAGCACTTAGAAAGAAGC \\
Ljwgs_044810.1 & LjLeuc & CCAGAGTTGTCAGAGTTGG & GACTAGCTAATCAAGTCCG \\
chr2.CM0201.55 & LjQOX & GCAACCTACTAGAAAGTGG & CAATTGATATGCTCATGGG \\
chr5.CM0909.44 & LjGST & GCATTCTATCGTTAGAACC & ACATCAAGAAGACAAACCCA \\
Ljwgs_075692.1.1 & LjHydr & GGATTCACACAACAGAAGC & CATAAAATGAATGGTCATGC \\
TC11891 & LjPI & GAAATGTGCAAGAAATTCC & AATAAAAGTCTCGCTATCTCC \\
B3IX38 & LjERF19 & TAGAGCCTACGATCGAGAAG & AACGACGAGTTGAACAGAG \\
chr1.CM1409.130.r2.d & GjMATE1 for & GGGGACAAGTTTGACAAAAAAC & GGGGACCACTTGTACAAGAAAGCT \\
chr1.CM1409.130.r2.d & GUS vector & GTGACAGTGCTTACATCG & GGGTTTCATTGTTGCCTTGTGT \\
GU441766 & LjMATE1 & GTATGGAGTGTTAAGATGCCC & TAACAAAGGTGACAAATCC \\
TC14054 & LjCCD7 & TAAAATGACTGCGTGGAAGC \\
chr1.CM0104.3050.r2.a & LjUBI & GCACCTTGTCTCACAGAAGAGACCC & AACAACAGAACACACAGACAATCC \\
LjSGA_008026.1 & LjVAP.a & CATGTAGAGGTTCTGAGG & AACAGAGTCACCAGAACC
\end{tabular}


non-parametric one-way ANOVA followed by Mann-Whitney Post hoc test.

\section{Biochemical Used for Bioassays}

Short-chain COs were purchased from Yaizu Suisankagaku Inudstry Corporation (Tokio, Japan). CO8 was kindly provided by Dr. Naoto Shibuya from Meiji University, Kawasaki, Japan to Andrea Genre. The preparation of solutions was performed following protocols indicated by Genre et al. (2013).

\section{Histochemical Analysis of Root Tissue}

Two thousand two hundred forty three base pairs upstream of LjMATE1 cds, one of the genes up-regulated in the microarray, was fused with the GUS gene in the vector pKGWFS70 in order to verify its activation at root level. The red fluorescent marker DsRED, under the control of the constitutive Arabidopsis Ubiquitin10 promoter (PUbq10; Limpens et al., 2005), was inserted. L. japonicus composite plants carrying transformed roots were treated with water or long chitooligosaccharides. Root fragments, showing DsRED fluorescence were selected under a stereomicroscope. After the treatment, the root segments were covered with freshly prepared GUS buffer $[0.1 \mathrm{mM}$ sodium phosphate buffer, $\mathrm{pH} 7,0.5 \mathrm{~mm} \mathrm{~K}_{4} \mathrm{Fe}(\mathrm{CN})_{6}, 5 \mathrm{mM}$ $\mathrm{K}_{3} \mathrm{Fe}(\mathrm{CN})_{6}, 0.3 \%$ Triton X, 0.3\% X-Glc]. Samples were incubated at $37^{\circ} \mathrm{C}$ for $16 \mathrm{~h}$ in the dark, washed with distilled water and observed under an optical microscope (Eclipse E400; Nikon).

\section{References}

Antolín-Llovera, M., Petutsching, E. K., Ried, M. K., Lipka, V., Nürnberger, T., Robatzek, S., et al. (2014). Knowing your friends and foes - plant receptor-like kinases as initiators of symbiosis or defence. New Phytol. 204, 791-802. doi: 10.1111/nph.13117

Boachon, B., Gamir, J., Pastor, V., Erb, M., Dean, J. V., Flors, V., et al. (2014). Role of two UDP-Glycosyltransferases from the L group of Arabidopsis in resistance against pseudomonas syringae. Eur. J. Plant Pathol. 139, 1-14. doi: 10.1007/s10658-014-0424-7

Bonfante, P., and Genre, A. (2015). Arbuscular mycorrhizal dialogues: do you speak "plantish" or "fungish"? Trends Plant Sci. 20, 150-154. doi: 10.1016/j.tplants.2014.12.002

Calcagno, C., Novero, M., Genre, A., Bonfante, P., and Lanfranco, L. (2012). The exudate from an arbuscular mycorrhizal fungus induces nitric oxide accumulation in Medicago truncatula roots. Mycorrhiza 22, 259-269. doi: 10.1007/s00572-011-0400-4

Cao, Y., Liang, Y., Tanaka, K., Nguyen, C. T., Jedrzejczak, R. P., Joachimiak, A., et al. (2014). The kinase LYK5 is a major chitin receptor in Arabidopsis and forms a chitin-induced complex with related kinase CERK1. eLife 3:e03766. doi: 10.7554/eLife.03766.

Chabaud, M., Genre, A., Sieberer, B. J., Faccio, A., Fournier, J., Novero, M., et al. (2011). Arbuscular mycorrhizal hyphopodia and germinated spore exudates trigger $\mathrm{Ca}^{2+}$ spiking in the legume and nonlegume root epidermis. New Phytologist. 189, 347-355. doi: 10.1111/j.1469-8137.2010. 03464.x

Czaja, L. F., Hogekamp, C., Lamm, P., Maillet, F., Martinez, E. A., Samain, E., et al. (2012). Transcriptional responses toward diffusible signals from symbiotic microbes reveal MtNFP- and MtDMI3-dependent reprogramming of host gene expression by arbuscular mycorrhizal fungal

\section{Author Contributions}

MG participated in the design of the experiment, carried out most of the experimental part and drafted the manuscript. AM produced exudates from $C$. trifolii and perform part of the qPCR and the GUS assay; MN followed the plant cultures, the spore germinating exudates production and the sampling for the microarray experiment; PB conceived the study, participated in its design and coordination and wrote the manuscript. All authors read and approved the final manuscript.

\section{Acknowledgments}

We thank Prof. Andrea Genre for sharing his knowledge about chitin oligosaccharides and for the critical reading of the manuscript, Dr. Mike Guether for his help with the first steps of the experiment, Dr. Matthew Hannah for the statistical analysis of the microarray data, Prof. Natalia Requena for kindly providing us with the GUS promoter vector containing the red root marker and Prof. Richard O'Connell for providing us C. trifolii strain. Financial support was provided by the National Project PRIN 2012 to PB. MG fellowship was funded by Fondazione Goria and Fondazione CRT.

\section{Supplementary Material}

The Supplementary Material for this article can be found online at: http://journal.frontiersin.org/article/10.3389/fpls.2015.00480

lipochitooligosaccharides. Plant Physiol. 159, 1671-1685. doi: 10.1104/pp.112. 195990

Deguchi, Y., Banba, M., Shimoda, Y., Chechetka, S. A., Suzuri, R., Okusako, Y., et al. (2007). Transcriptome profiling of Lotus japonicus roots during arbuscular mycorrhiza development and comparison with that of nodulation. DNA Res. 14, 117-133. doi: 10.1093/dnares/dsm014

Dodds, P. N., and Rathjen, J. P. (2010). Plant immunity: towards an integrated view of plant-pathogen interactions. Nat. Rev. Genet. 11, 539-548. doi: $10.1038 / \mathrm{nrg} 2812$

Doss, R. P. (2005). Treatment of pea pods with Bruchin B results in upregulation of a gene similar to MtN19. Plant Physiol. Biochem. 43, 225-231. doi: 10.1016/j.plaphy.2005.01.016

Enyedi, A. J., and Raskin, I. (1993). Induction of UDP-glucose:salicylic acid glucosyltransferase activity in tobacco mosaic Virus-inoculated tobacco (Nicotiana tabacum) leaves. Plant Physiol. 101, 1375-1380. doi: 10.1104/pp.101.4.1375

Evangelisti, E., Rey, T., and Schornack, S. (2014). Cross-interference of plant development and plant-microbe interactions. Curr. Opin. Plant Biol. 20, 118126. doi: 10.1016/j.pbi.2014.05.014

Fiorilli, V., Catoni, M., Miozzi, L., Novero, M., Accotto, G. P., and Lanfranco, L. (2009). Global and cell-type gene expression profiles in tomato plants colonized by an arbuscular mycorrhizal fungus. New Phytologist 184, 975-987. doi: 10.1111/j.1469-8137.2009.03031.x

Floss, D. S., Levy, J. G., Lévesque-Tremblay, V., Pumplin, N., and Harrison, M. J. (2013). DELLA proteins regulate arbuscule formation in arbuscular mycorrhizal symbiosis. Proc. Natl. Acad. Sci. U.S.A. 51:110. doi: $10.1073 /$ pnas. 1308973110

Gage, D. J. (2004). Infection and invasion of roots by symbiotic, nitrogen-fixing rhizobia during nodulation of temperate legumes. Microbiol. Mol. Biol. Rev. 68, 280-300. doi: 10.1128/MMBR.68.2.280-300.2004 
Gaude, N., Bortfeld, S., Duensing, N., Lohse, M., and Krajinski, F. (2012). Arbuscule-containing and non-colonized cortical cells of mycorrhizal roots undergo extensive and specific reprogramming during arbuscular mycorrhizal development. Plant J. 69, 510-528. doi: 10.1111/j.1365-313X.2011.04810.x

Gautier, L., Cope, L., Bolstad, B. M., and Irizarry, R. A. (2004). affy-analysis of Affymetrix GeneChip data at the probe level. Bioinformatics 20, 307-315. doi: 10.1093/bioinformatics/btg405

Genre, A., Chabaud, M., Balzergue, C., Puech-Pagès, V., Novero, M., Rey, T., et al. (2013). Short-chain chitin oligomers from arbuscular mycorrhizal fungi trigger nuclear $\mathrm{Ca}^{2+}$ spiking in Medicago truncatula roots and their production is enhanced by strigolactone. New Phytol. 198, 190-202. doi: 10.1111/nph.12146

Genre, A., Ortu, G., Bertoldo, C., Martino, E., and Bonfante, P. (2009). Biotic and abiotic stimulation of root epidermal cells reveals common and specific responses to arbuscular mycorrhizal fungi. Plant Physiol. 149, 1424-1434. doi: 10.1104/pp.108.132225

Gentleman, R. C., Carey, V. J., Bates, D. M., Bolstad, B., Dettling, M., Dudoit, S., et al. (2004). Bioconductor: open software development for computational biology and bioinformatics. Genome Biol. 5:R80. doi: 10.1186/gb-2004-5$10-r 80$

Gobbato, E., Marsh, J. F., Vernié, T., Wang, E., Maillet, F., Kim, J., et al. (2012). A GRAS-type transcription factor with a specific function in mycorrhizal signaling. Curr. Biol. 22, 2236-2241. doi: 10.1016/j.cub.2012.09.044

González-Guerrero, M., Matthiadis, A., Saez, Á., and Long, T. A. (2014). Fixating on metals: new insights into the role of metals in nodulation and symbiotic nitrogen fixation. Front. Plant Sci. 5:45. doi: 10.3389/fpls.2014.00045

Gough, C., and Jacquet, C. (2013). Nod factor perception protein carries weight in biotic interactions. Trends Plant Sci. 18, 566-574. doi: 10.1016/j.tplants.2013.06.001

Gourion, B., Berrabah, F., Ratet, P., and Stacey, G. (2015). Rhizobium-legume symbioses: the crucial role of plant immunity. Trends Plant Sci. 20, 186-194. doi: 10.1016/j.tplants.2014.11.008

Guether, M., Balestrini, R., Hannah, M., He, J., Udvardi, M. K., and Bonfante, P. (2009). Genome-wide reprogramming of regulatory networks, transport, cell wall and membrane biogenesis during arbuscular mycorrhizal symbiosis in Lotus japonicus. New Phytol. 182, 200-212. doi: 10.1111/j.14698137.2008.02725.x

Gutjahr, C., and Parniske, M. (2013). Cell and developmental biology of arbuscular mycorrhiza symbiosis. Annu. Rev. Cell Dev. Biol. 29, 593-617. doi: 10.1146/annurev-cellbio-101512-122413

Handa, Y., Nishide, H., Takeda, N., Suuki, Y., Kawaguchi, M., and Saito, K. (2015). RNA-seq transcriptional profiling of an arbuscular mycorrhiza provides insights into regulated and coordinated gene expression in Lotus japonicus and Rhizophagus irregularis. Plant Cell Physiol. doi: 10.1093/pcp/pcv071 [Epub ahead of print].

Hao, Z., Fayolle, L., van Tuinen, D., Chatagnier, O., Li, X., Gianinazzi, S., et al. (2012). Local and systemic mycorrhiza-induced protection against the ectoparasitic nematode Xiphinema index involves priming of defence gene responses in grapevine. J. Exp. Bot. 63, 3657-3672. doi: 10.1093/jxb/ ers046

Hayafune, M., Berisio, R., Marchetti, R., Silipo, A., Kayama, M., Desaki, Y., et al. (2014). Chitin-induced activation of immune signaling by the rice receptor CEBiP relies on a unique sandwich-type dimerization. Proc. Natl. Acad. Sci. U.S.A. 111, E404-E413. doi: 10.1073/pnas.1312099111

Hayashi, M., and Parniske, M. (2014). Symbiosis and pathogenesis: what determines the difference? Curr. Opin. Plant Biol. 20:v-vi. doi: 10.1016/j.pbi.2014.07.008

Hogekamp, C., Arndt, D., Pereira, P. A., Becker, J. D., Hohnjec, N., and Küster, H. (2011). Laser microdissection unravels cell-type-specific transcription in arbuscular mycorrhizal roots, including caat-box transcription factor gene expression correlating with fungal contact and spread. Plant Physiol. 157, 2023-2043. doi: 10.1104/pp.111.186635

Hogekamp, C., and Küster, H. (2013). A roadmap of cell-type specific gene expression during sequential stages of the arbuscular mycorrhiza symbiosis. BMC Genomics 14:306. doi: 10.1186/1471-2164-14-306

Irizarry, R. A., Hobbs, B., Collin, F., Beazer-Barclay, Y. D., Antonellis, K. J., Scherf, U., et al. (2003). Exploration, normalization, and summaries of high density oligonucleotide array probe level data. Biostatistics 4, 249-264. doi: 10.1093/biostatistics/4.2.249
Jones, J. D. G., and Dangl, J. L. (2006). The plant immune system. Nature 444, 323-329. doi: 10.1038/nature05286

Kiers, E. T., Duhamel, M., Beesetty, Y., Mensah, J. A., Franken, O., Verbruggen, E., et al. (2011). Reciprocal rewards stabilize cooperation in the mycorrhizal symbiosis. Science 333, 880-882. doi: 10.1126/science.1208473

Kloppholz, S., Kuhn, H., and Requena, N. (2011). A secreted fungal effector of Glomus intraradices promotes symbiotic biotrophy. Curr. Biol. 21, 1204-1209. doi: 10.1016/j.cub.2011.06.044

Liang, Y., Cao, Y., Tanaka, K., Thibivilliers, S., Wan, J., Choi, J., et al. (2013). Nonlegumes respond to rhizobial nod factors by suppressing the innate immune response. Science 341, 1384-1387. doi: 10.1126/science.12 42736

Liang, Y., Tóth, K., Cao, Y., Tanaka, K., Espinoza, C., and Stacey, G. (2014). Lipochitooligosaccharide recognition: an ancient story. New Phytol. 204, 289 296. doi: 10.1111/nph.12898

Libault, M., Farmer, A., Brechenmacher, L., Drnevich, J., Langley, R. J., Bilgin, D. D., et al. (2010). Complete transcriptome of the soybean root hair cell, a single-cell model, and its alteration in response to Bradyrhizobium japonicum infection. Plant Physiol. 152, 541-552. doi: 10.1104/pp.109. 148379

Limpens, E., Mirabella, R., Fedorova, E., Franken, C., Franssen, H., Bisseling, T., et al. (2005). Formation of organelle-like N2-fixing symbiosomes in legume root nodules is controlled by DMI2. Proc. Natl. Acad. Sci. U.S.A. 102, 10375-10380. doi: 10.1073/pnas.0504284102

Macho, A. P., and Zipfel, C. (2014). Plant PRRs and the activation of innate immune signaling. Mol. Cell 54, 263-272. doi: 10.1016/j.molcel.2014.03.028

Maillet, F., Poinsot, V., André, O., Puech-Pagès, V., Haouy, A., Gueunier, M., et al. (2011). Fungal lipochitooligosaccharide symbiotic signals in arbuscular mycorrhiza. Nature 469, 58-63. doi: 10.1038/nature09622

Marino, D., Andrio, E., Danchin, E. G. J., Oger, E., Gucciardo, S., Lambert, A., et al. (2011). A Medicago truncatula NADPH oxidase is involved in symbiotic nodule functioning. New Phytol. 189, 580-592. doi: 10.1111/j.1469-8137.2010. 03509.x

Miyata, K., Kozaki, T., Kouzai, Y., Ozawa, K., Ishii, K., Asamizu, E., et al. (2014). The bifunctional plant receptor, OsCERK1, regulates both chitin-triggered immunity and arbuscular mycorrhizal symbiosis in rice. Plant Cell Physiol. 55, 1864-1872. doi: 10.1093/pcp/pcu129

Moreau, S., Verdenaud, M., Ott, T., Letort, S., de Billy, F., Niebel, A., et al. (2011). Transcription reprogramming during root nodule development in Medicago truncatula. PLoS ONE 6:e16463. doi: 10.1371/journal.pone.0016463

Naya, L., Paul, S., Valdés-López, O., Mendoza-Soto, A. B., Nova-Franco, B., Sosa-Valencia, G., et al. (2014). Regulation of copper homeostasis and biotic interactions by microrna $398 \mathrm{~b}$ in common bean. PLOS ONE 9:e84416. doi: 10.1371/journal.pone.0084416

Oldroyd, G. E. D. (2013). Speak, friend, and enter: signaling systems that promote beneficial symbiotic associations in plants. Nat. Rev. Microbiol. 11, 252-263. doi: $10.1038 /$ nrmicro2990

Parniske, M. (2008). Arbuscular mycorrhiza: the mother of plant root endosymbioses. Nat. Rev. Micro. 6, 763-775. doi: 10.1038/nrmicro1987

Pietraszewska-Bogiel, A., Lefebvre, B., Koini, M. A., Klaus-Heisen, D., Takken, F. L. W., Geurts, R., et al. (2013). Interaction of Medicago truncatula lysin motif receptor-like kinases, NFP and LYK3, produced in Nicotiana benthamiana induces defence-like responses. PLoS ONE 8:e65055. doi: 10.1371/journal.pone.0065055

Pumplin, N., Mondo, S. J., Topp, S., Starker, C. G., Gantt, J. S., and Harrison, M. J. (2009). Medicago truncatula Vapyrin is a novel protein required for arbuscular mycorrhizal symbiosis. Plant J. 61, 482-494. doi: 10.1111/j.1365313X.2009.04072.x

Rey, T., Chatterjee, A., Buttay, M., Toulotte, J., and Schornack, S. (2015). Medicago truncatula symbiosis mutants affected in the interaction with a biotrophic root pathogen. New Phytol. 206, 497-500. doi: 10.1111/nph.13233

Rey, T., Nars, A., Bonhomme, M., Bottin, A., Huguet, S., Balzergue, S., et al. (2013). NFP, a LysM protein controlling Nod factor perception, also intervenes in Medicago truncatula resistance to pathogens. New Phytol. 198, 875-886. doi: 10.1111/nph.12198

Rey, T., and Schornack, S. (2013). Interactions of beneficial and detrimental rootcolonizing filamentous microbes with plant hosts. Genome Biol. 14:121. doi: 10.1186/gb-2013-14-6-121 
Ried, M. K., Antolín-Llovera, M., and Parniske, M. (2014). Spontaneous symbiotic reprogramming of plant roots triggered by receptor-like kinases. eLife 3:e03891. doi: 10.7554/eLife.03891

Ririe, K. M., Rasmussen, R. P., and Wittwer, C. T. (1997). Product differentiation by analysis of DNA melting curves during the polymerase chain reaction. Anal. Biochem. 245, 154-160. doi: 10.1006/abio.1996.9916

Ruzicka, D., Chamala, S., Barrios-Masias, F. H., Martin, F., Smith, S., Jackson, L. E., et al. (2013). Inside arbuscular mycorrhizal roots molecular probes to understand the symbiosis. Plant Genome 6, 1-13. doi: 10.3835/plantgenome2012.06.0007

Sanchez-Vallet, A., Saleem-Batcha, R., Kombrink, A., Hansen, G., Valkenburg, D.-J., Thomma, B. P., et al. (2013). Fungal effector Ecp6 outcompetes host immune receptor for chitin binding through intrachain LysM dimerization. eLife 2:e00790. doi: 10.7554/eLife.00790

Smyth, G. K. (2004). Linear models and empirical Bayes methods for assessing differential expression in microarray experiments. Stat. Appl. Genet. Mol. Biol. 3, 1-25. doi: 10.2202/1544Ü6115.1027

Sun, J., Miller, J. B., Granqvist, E., Wiley-Kalil, A., Gobbato, E., Maillet, F., et al. (2015). Activation of symbiosis signaling by arbuscular mycorrhizal fungi in legumes and rice. Plant Cell 3, 823-838. doi: 10.1105/tpc.114.131326

Takanashi, K., Yokosho, K., Saeki, K., Sugiyama, A., Sato, S., Tabata, S., et al. (2013). LjMATE1: a citrate transporter responsible for iron supply to the nodule infection zone of Lotus japonicus. Plant Cell Physiol. 54, 585-594. doi: $10.1093 / \mathrm{pcp} / \mathrm{pct} 019$

Tisserant, E., Malbreil, M., Kuo, A., Kohler, A., Symeonidi, A., Balestrini, R., et al. (2013). Genome of an arbuscular mycorrhizal fungus provides insight into the oldest plant symbiosis. Proc. Natl. Acad. Sci. U.S.A. 110, 20117-20122. doi: 10.1073/pnas.1313452110

Torregrosa, C., Cluzet, S., Fournier, J., Huguet, T., Gamas, P., Prospéri, J.-M., et al. (2004). Cytological, genetic, and molecular analysis to characterize compatible and incompatible interactions between Medicago truncatula and Colletotrichum trifolii. Mol. Plant Microbe Interact. 17, 909-920. doi: 10.1094/MPMI.2004.17.8.909
Wang, E., Schornack, S., Marsh, J. F., Gobbato, E., Schwessinger, B., Eastmond, P., et al. (2012). A common signaling process that promotes mycorrhizal and oomycete colonization of plants. Curr. Biol. 22, 2242-2246. doi: 10.1016/j.cub.2012.09.043

Wang, W., Xie, Z.-P., and Staehelin, C. (2014). Functional analysis of chimeric lysin motif domain receptors mediating Nod factor-induced defense signaling in Arabidopsis thaliana and chitin-induced nodulation signaling in Lotus japonicus. Plant J. 78, 56-69. doi: 10.1111/tpj.12450

Wulf, A., Manthey, K., Doll, J., Perlick, A. M., Linke, B., Bekel, T., et al. (2003). Transcriptional changes in response to arbuscular mycorrhiza development in the model plant Medicago truncatula. Mol. Plant Microbe Interact. 16, 306-314. doi: 10.1094/MPMI.2003.16.4.306

Zhang, X., Dong, W., Sun, J., Feng, F., Deng, Y., He, Z., et al. (2015). The receptor kinase CERK1 has dual functions in symbiosis and immunity signalling. Plant J. 81, 258-267. doi: 10.1111/tpj.12723

Zouari, I., Salvioli, A., Chialva, M., Novero, M., Miozzi, L., Tenore, G. C., et al. (2014). From root to fruit: RNA-Seq analysis shows that arbuscular mycorrhizal symbiosis may affect tomato fruit metabolism. BMC Genomics 15:221. doi: 10.1186/1471-2164-15-221

Zurbriggen, M. D., Carrillo, N., and Hajirezaei, M.-R. (2010). ROS signaling in the hypersensitive response. Plant Signal. Behav. 5, 393-396. doi: $10.4161 /$ psb.5.4.10793

Conflict of Interest Statement: The authors declare that the research was conducted in the absence of any commercial or financial relationships that could be construed as a potential conflict of interest.

Copyright () 2015 Giovannetti, Mari, Novero and Bonfante. This is an open-access article distributed under the terms of the Creative Commons Attribution License (CC BY). The use, distribution or reproduction in other forums is permitted, provided the original author(s) or licensor are credited and that the original publication in this journal is cited, in accordance with accepted academic practice. No use, distribution or reproduction is permitted which does not comply with these terms. 